\title{
SATISFACCIÓN DE USUARIOS HOSPITALIZADOS EN UN HOSPITAL DE APURIMMAC, PERÚ
}

\author{
Elsa Yudy Sihuin-Tapia ${ }^{1, a}$, Oscar Elisban Gómez-Quispe ${ }^{2, b}, V$ Vladimiro Ibáñez-Quispe ${ }^{3, c}$
}

\begin{abstract}
RESUMEN
Con el objeto de determinar la satisfacción en los usuarios hospitalizados en el Hospital Subregional de Andahuaylas se encuestó a una muestra de 175 usuarios utilizando el modelo multidimensional Servqual. La estimación de variables asociadas con la satisfacción de los usuarios hospitalizados fue realizada a través de análisis bivariado y multivariable empleando regresión logística. Encontramos 25,0\% de satisfacción. Estuvieron asociados a la baja satisfacción en lo usuarios el tener nivel de estudios secundaria (ORa: 0,05; IC 95\%: 0,01-0,64) y haber sido hospitalizado en el servicio de cirugía (ORa 0,14; IC 95\%: 0,04-0,53). Se concluye que existió baja satisfacción con la calidad del servicio recibido por los usuarios hospitalizados, y esta estuvo asociada al nivel de educación y al tipo de servicio de hospitalización.
\end{abstract}

Palabras clave: Satisfacción del paciente; Calidad de la atención de salud; Hospitales públicos (fuente: DeCS BIREME).

\section{SATISFACTION OF HOSPITALIZED PATIENTS IN A HOSPITAL IN APURIMAC, PERU}

\begin{abstract}
In order to determine the satisfaction of hospitalized patients in the Sub-regional Hospital of Andahuaylas, 175 patients were surveyed using the Servqual multidimensional model. The estimate of variables associated with the satisfaction of the hospitalized patients was performed by using bivariate and multivariate logistic regression analysis. We found $25.0 \%$ satisfaction. Lower levels of satisfaction were associated with having a secondary level education (aOR: $0.05 ; 95 \% \mathrm{Cl}$ : 0.01 to 0.64 ) and with having been hospitalized in the surgery department (aOR $0.14, \mathrm{Cl}: 95 \%: 0.04$ to 0.53 ). It was concluded that there was a low level of satisfaction with the quality of care received by hospitalized patients and this was associated with the level of education and type of hospital department.
\end{abstract}

Key words: Patient satisfaction; Quality of health care; Hospitals public (source: MeSH NLM).

\section{INTRODUCCIÓN}

El servicio de salud que se brinda en hospitales públicos es muy complejo debido a los diversos procesos y procedimientos que se manejan. Un objetivo primordial en estos centros es conocer la calidad del servicio que han recibido los usuarios. En un entorno dinámico en el que hay cambios continuos en la demografía, las preferencias y los estilos de vida, estas evaluaciones de calidad se han convertido en esencial para los responsables de la gestión de hospitales, porque permite entender las brechas del servicio entre las expectativas y percepciones de los usuarios, siendo importante para introducir estrategias de mejora. ${ }^{(1-3)}$.
Para determinar el grado de satisfacción de usuarios en los establecimientos de salud, se ha aplicado el modelo multidimensional Servqual, que tiene elevada confiabilidad y validez ${ }^{(4)}$. Este instrumento de medición contiene uno de los cuestionarios más empleados para medir la calidad de los usuarios. Intervienen fundamentalmente dos factores, las expectativas y las percepciones, la diferencia entre ambas da como resultado el grado de satisfacción o insatisfacción ${ }^{(5,6)}$; esta es la conformidad expresada por el usuario en función de su expectativa de la atención recibida.

Esta metodología evalúa la satisfacción de los usuarios en cinco atributos o dimensiones del servicio como:

\footnotetext{
Hospital Subregional de Andahuaylas. Apurímac, Perú.

Facultad de Medicina Veterinaria y Zootecnia. Universidad Nacional Micaela Bastidas de Apurímac. Apurímac, Perú.

Facultad de Ingeniería Estadística e Informática. Universidad Nacional del Altiplano. Puno, Perú.

Licenciada en Enfermería; magíster en Administración de la Salud; ${ }^{b}$ médico veterinario, magíster en Reproducción animal; ingeniero economista; ingeniero estadístico, magíster en Informática, doctor en Administración.

Recibido: : 04-03-14 Aprobado: 04-03-15
}

Citar como: Sihuin-Tapia EY, Gómez-Quispe OE, Ibáñez-Quispe V. Satisfacción de usuarios hospitalizados en un hospital de Apurímac, Perú. Rev Peru Med Exp Salud Publica. 2015;32(2):299-302. 
fiabilidad o la habilidad y el cuidado en brindar el servicio ofrecido; la capacidad de respuesta o la disposición y la buena voluntad de ayudar a los usuarios y proveerlos de un servicio rápido y oportuno; la seguridad o la cortesía, la habilidad para transmitir credibilidad, confianza y confidencia en la atención con inexistencia de peligros, riesgos o dudas; empatía o la disponibilidad para pensar primero en el paciente y atender según características y situaciones particulares, cuidado y atención individualizada; así como aspectos tangibles o la apariencia de las instalaciones físicas, equipamiento, apariencia del personal y materiales de comunicación ${ }^{(7)}$.

El Ministerio de Salud (MINSA) del Perú reconoce que, la calidad en la prestación de salud es una preocupación fundamental en la gestión del sector, e indica que el grado de calidad es la medida en que se espera que la atención suministrada logre el equilibrio más favorable de riesgos y beneficios para el usuario ${ }^{(8)}$. En el marco de estas consideraciones, el Hospital Subregional de Andahuaylas, -hospital tipo II-, localizado en la provincia de Andahuaylas en la sierra centro sur del Perú, se realizan muchas y diversas atenciones de salud a usuarios en los diferentes servicios de hospitalización; sin embargo, existen usuarios hospitalizados que al ser dados de alta muestran cierta insatisfacción, debido a que el grado de exigencia actual de los pacientes y de sus familiares se ha incrementado, por el incremento de la información y, con ello, también de sus expectativas. El objeto del estudio fue determinar el nivel de satisfacción de los usuarios hospitalizados en el Hospital Subregional de Andahuaylas.

\section{EL ESTUDIO}

La investigación fue de tipo relacional transversal, desarrollada durante el primer semestre del 2011. Se seleccionó una muestra de 175 usuarios de una población de 320 , de promedio mensual, dados de alta en los servicios de hospitalización de Cirugía, Ginecología, Medicina y Traumatología en el Hospital Subregional de Andahuaylas, en la región de Apurimac, Perú. Los criterios de inclusión fueron: usuarios dados de alta poshospitalización, de ambos sexos, de 18 a 80 años de edad, con estado de conciencia (lúcido, orientado en tiempo y espacio), que accedan a participar en la entrevista y con tiempo de hospitalización mínima de tres días.

Previamente, se coordinó con los responsables de los servicios de hospitalización, y se entrenó a encuestadores externos en la metodología Servqual. La toma de datos tuvo carácter anónimo, y los pacientes fueron informados previamente obteniéndose el consentimiento informado.
La satisfacción de usuarios según dimensiones de calidad, se estimó con el análisis multidimensional Servqual, que evalúa la diferencia o brecha entre las expectativas y la percepción de usuarios en cinco dimensiones ${ }^{(7)}$, a través de un total de 22 pares de preguntas acerca de la calidad del servicio; en donde una brecha positiva sugiere que las expectativas se han cumplido o se han excedido, mientras que una brecha negativa indica una deficiencia o insatisfacción ${ }^{(9)}$.

La estimación de variables asociadas a la satisfacción de los usuarios hospitalizados fue realizado a través de análisis bivariado y multivariable empleando la regresión logística con el software estadístico SPSS v.18,0, donde el nivel de estudios, el servicio de hospitalización, el tiempo de hospitalización, el tipo de seguro, el grupo etario, el sexo y la condición del encuestado, fueron considerados variables independientes, y la satisfacción del usuario como variable dependiente.

\section{HALLAZGOS}

El $60 \%$ de los encuestados eran de sexo masculino, el $4,0 \%$ eran adultos mayores, el $36,0 \%$ de los usuarios tenían nivel educativo primario, el 6,3\% se hospitalizaron

Tabla 1. Frecuencia de características de los usuarios hospitalizados en el Hospital Subregional de Andahuaylas, 2011

\begin{tabular}{|c|c|c|}
\hline Variables & $\mathbf{n}$ & $(\%)$ \\
\hline \multicolumn{3}{|l|}{ Sexo } \\
\hline Femenino & 70 & $(40,0)$ \\
\hline Masculino & 105 & $(60,0)$ \\
\hline \multicolumn{3}{|l|}{ Grupo etario } \\
\hline Adulto & 168 & $(96,0)$ \\
\hline Adulto mayor & 7 & $(4,0)$ \\
\hline \multicolumn{3}{|l|}{ Nivel de estudios } \\
\hline Ninguno & 46 & $(26,3)$ \\
\hline Primaria & 63 & $(36,0)$ \\
\hline Secundaria & 62 & $(35,4)$ \\
\hline Superior & 4 & $(2,3)$ \\
\hline \multicolumn{3}{|l|}{ Servicio de hospitalización } \\
\hline Cirugía & 70 & $(40,0)$ \\
\hline Ginecología & 35 & $(20,0)$ \\
\hline Medicina & 35 & $(20,0)$ \\
\hline Traumatología & 35 & $(20,0)$ \\
\hline \multicolumn{3}{|l|}{ Tiempo de hospitalización } \\
\hline Corto & 70 & $(40,0)$ \\
\hline Mediano & 94 & $(53,7)$ \\
\hline Prolongado & 11 & $(6,3)$ \\
\hline \multicolumn{3}{|l|}{ Tipo de seguro } \\
\hline Ninguno & 9 & $(5,1)$ \\
\hline SIS & 160 & $(91,4)$ \\
\hline SOAT & 6 & $(3,4)$ \\
\hline \multicolumn{3}{|l|}{ Condición del encuestado } \\
\hline Acompañante & 27 & $(15,4$ \\
\hline Paciente & 148 & $(84,6)$ \\
\hline
\end{tabular}




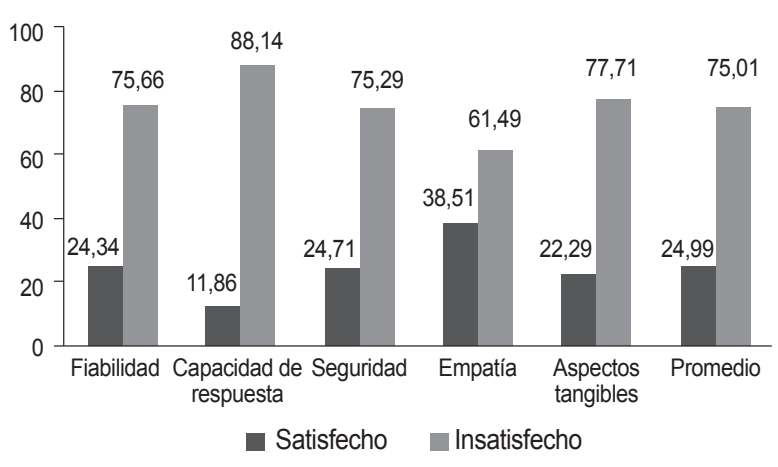

Figura 1. Satisfacción por dimensiones según Servqual de los usuarios hospitalizados en el Hospital Subregional de Andahuaylas, 2011

por tiempo prolongado, y el $91,4 \%$ de usuarios se atendieron por el Sistema Integral de Salud (SIS).

Respecto a la satisfacción de los usuarios hospitalizados, se encontró como promedio una satisfacción del 25,0\%. La satisfacción según las dimensiones de fiabilidad, capacidad de respuesta, seguridad, empatía y aspectos tangibles estuvieron entre 11,9 a $38,5 \%$ (Figura 1).

En el análisis bivariado, se encontró menor probabilidad para la satisfacción de los usuarios hospitalizados con las variables nivel de estudios secundaria (OR 0,03; IC 95\%: 0,01-0,38; $p=0,001)$ y haber sido hospitalizado en el servicio de cirugía (OR 0,16; IC 95\%: 0,05$0,62 ; p=0,001)$; en el análisis multivariable el nivel de estudios secundaria (ORa: 0,05; IC 95\%: 0,01-0,64; $p=0,021)$ y hospitalización en el servicio de cirugía (ORa 0,14; IC 95\%: 0,04-0,53; $p=0,004$ ) permanecieron como variables que afectan la satisfacción del usuario ajustado por tiempo de hospitalización, tipo de seguro, grupo etario, sexo y condición del encuestado.

\section{DISCUSIÓN}

Se elaboró un reporte sobre satisfacción de usuarios hospitalizados medido por la metodología Servqual en este hospital del Perú. La satisfacción global de usuarios de este estudio fue inferior al hallado en pacientes hospitalizados donde se encuentran hasta una satisfacción promedio del $52,2 \%$ en los pacientes dados de alta ${ }^{(13)}$. Al respecto, la información disponible sobre satisfacción de usuarios hospitalizados es muy limitada. La medición de la calidad a través de la metodología Servqual mayormente se ha empleado en pacientes ambulatorios o de consulta externa, en este contexto, en el servicio de consultas externa de medicina interna del Hospital General de Lima (Perú) se encontró que la satisfacción fue del 44,4\% ${ }^{(11)}$; con la misma metodología, pero en usuarios del servicio ambulatorio, en el Hospital Nacional Cayetano Heredia en Lima, se reportó un $46,8 \%$ de satisfacción en usuarios de consulta externa y $52,9 \%$ en el servicio de emergencia (12); en otro estudio realizado en 15 hospitales ${ }^{(14)}$ en un estado de México se encontró que la satisfacción del estudio fue $84,9 \%$, aunque en esta investigación no se utilizó la metodología del Servqual, sino el cuestionario diseñado mediante las bases metodológicas y conceptuales de Avedis Donabedian ${ }^{(15)}$ que contenían 69 preguntas que abarcaban seis dimensiones de la calidad.

La satisfacción encontrada fue inferior a la informada en el servicio de consulta externa de medicina interna del Hospital Nacional Arzobispo Loayza de Lima(11), utilizando la encuesta Servqual modificado, siendo la satisfacción por dimensiones del $47,6,44,8,56,1,52,4$ y $58,5 \%$ para aspectos tangibles, confiabilidad, respuesta rápida, seguridad y empatía, respectivamente. En un estudio realizado por Yesilada y Direktör ${ }^{(16)}$, en los hospitales públicos y privados de Turquía, de las cinco dimensiones del Servqual, propuestos por Parasuraman ${ }^{(17)}$, solo se pudo validar la fiabilidad, empatía y aspectos tangibles, considerando solo estas como dimensiones del servicio de calidad.

En el estudio, estuvieron asociadas las variables nivel de estudio y el servicio de hospitalización, lo que concuerda con lo reportado en el servicio de consulta externa de medicina interna del Hospital Nacional Arzobispo Loayza de Lima, Perú(11), donde utilizando la encuesta Servqual, la satisfacción global estuvo asociado al nivel de instrucción. Similarmente, Uzun, al estudiar la satisfacción de la atención de enfermería en un hospital de Turquía, utilizando Servqual, encontró que los pacientes más jóvenes, hombres y con menor nivel de educación evaluaron más negativamente la calidad asistencial ${ }^{(18)}$, y en otro estudio realizado por Bakar et al., en un Hospital Universitario de Turquía, con la misma metodología, encontraron que los pacientes jóvenes y con un mayor nivel de estudios puntuaron más bajo la calidad asistencial (19). Dichos factores se comportaban de manera particular según el tipo de establecimiento de salud, debido fundamentalmente a la población usuaria distinta, relativizando la comparación del nivel de satisfacción y de sus factores asociados entre tales establecimientos ${ }^{(20)}$.

Los valores de los usuarios influirían en el grado de satisfacción, inclusive es diferente el grado de satisfacción de un cliente que acude al hospital por primera vez que aquel paciente que acude en forma continua; posiblemente las consideraciones éticas o morales estén incorporadas dentro de las necesidades y expectativas del cliente ya que, indudablemente, estas van a ser diferentes dependiendo de la religión que profesan, creencias y tradiciones o de las normas éticas que rigen la vida del cliente ${ }^{(6)}$. 
Entre las principales limitaciones de este estudio estarían, el no realizar la validación previa de esta metodología para evaluar la calidad en la atención de usuarios hospitalizados así como la alta frecuencia de pacientes sin nivel educativo, sin embargo, esto fue controlado a través del entrenamiento a los encuestadores en el manejo de la metodología Servqual.

Se concluye que existe baja satisfacción de usuarios hospitalizados, este comportamiento es similar en los niveles de las dimensiones de calidad. El nivel de educación y el tipo de servicio de hospitalización fueron considerados variables asociadas a la baja satisfacción de usuarios hospitalizados.

Contribuciones de autoría: EYST y OEGQ participaron en la concepción del estudio, análisis y redacción del artículo, EYST participó en la recolección de resultados VIQ participó en el análisis de datos y discusión del artículo.

Fuentes de financiamiento: autofinanciado.

Conflictos de interés: los autores manifiestan no tener conflictos de interés.

\section{REFERENCIAS BIBLIOGRÁFICAS}

1. Babakus E, Mangold WG. Adapting the SERVQUAL scale to hospital services: an empirical investigation. Health Serv Res. 1992 Feb;26:767-86.

2. Regaira E, Sola M, Goñi R, Del Barrio M, Margall MA, Asiain MC. La calidad asistencial en cuidados intensivos evaluada por los pacientes mediante la escala SERVQUAL. Enferm Intensiva. 2010;21(1):3-10.

3. Chakravarty A. Evaluation of service quality of hospital outpatient department services. Medical Journal Armed Forces India. 2011;67(3):2214.

4. Lam SK. SERVQUAL: A tool for measuring patient's opinions of hospital service quality in Hong Kong. Total Quality Management. 2001;8(4):14552.

5. Fatma Pakdil F, Harwood TN Patient satisfaction in a preoperative assessment clinic: an analysis using SERVQUAL dimensions. Total Quality Management \& Bussiness Excellence. 2005;16(1):15-30.

6. Miyahira J. Calidad en los servicios de salud ¿Es posible? Rev Med Hered. 2001;12(3):76-7.

7. Parasuraman A, Zeithaml VA, Berry LL. SERVQUAL: A Multiple Item Scale for Measuring Consumer Perceptions of Service Quality. J Retailing.1988;64(1):12-40.

8. Perú, Ministerio de Salud. Sistema de Gestión de la Calidad en Salud: Documento técnico RM 519-2006
[Internet]. Lima: MINSA; 2007 [citado el 19 de febrero de 2014]. Disponible en:

http://www.minsa.gob.pe/dgsp/archivo/ SistemaGestiondelaCalidad.pdf

9. Lorin V, Raluca I, Madalina C. The Assessment of Perceived Service Quality of Public Health Care Services in Romania Using the SERVQUAL Scale. Procedia Economics and Finance. 2013;6:573-85.

10. Mira JJ, Buil JA, Rodríguez-Marín J, Aranaz J. Calidad percibida del cuidado hospitalario. Gac Sanit. 1997;11(4):176-89.

11. Casalino-Carpio GE. Calidad de servicio de la consulta externa de Medicina Interna de un hospital general de Lima mediante la encuesta Servqual. Rev Soc Peru Med Interna. 2008;21(4):143-52.

12. Cabello E, Chirinos JL. Validación y aplicabilidad de encuestas SERVQUAL modificadas para medir la satisfacción de usuarios externos en servicios de salud. Rev Med Hered. 2012;23(2):88-95.

13. Gómez-Payo S, Del Río-Moro O, Manrique-García A, RodríguezMartín MC, Fernández-Pardo MJ. 2012. Satisfacción del usuario en el Hospital Geriátrico Virgen del Valle de Toledo. Revista de Calidad Asistencial. 2012;27(2):78-84

14. Ortiz RM, Muñoz $S$, Torres E. Satisfacción de los usuarios de 15 hospitales de Hidalgo, México. Rev Esp Salud Publica. 2004;78(4):527-37.
15. Donabedian A. Evaluating the quality of medical care. 1966. Milbank Q. 2005;83(4):691-729.

16. Yesilada F, Direktör E. Health care service quality: A comparison of public and private hospitals. Afr J Bus Manag. 2010;4(6):962-71.

17. Parasuraman A, Berry LL, Zeithaml V. More on Improving Service Quality Measurement. J Retailing.1993;69(1):140-7.

18. Uzun O. Patient satisfaction with nursing care at a university hospital in Turkey. J Nurs Care Qual. 2001 Oct;16(1):24-33.

19. Bakar C, Akgün HS, Al Assaf AF. The role of expectations in patient assessments of hospital care: an example from a university hospital network, Turkey. Int J Health Care Qual Assur. 2008;21(4):343-55.

20. Seclén-Palacin J, Darras Ch. Satisfacción de usuarios de los servicios de salud: Factores sociodemográficos y de accesibilidad asociados. Perú, 2000. An Fac Med Lima. 2005;66(2)127-41.

Correspondencia: Oscar Elisban Gómez Quispe

Dirección: Facultad de Medicina Veterinaria y Zootecnia, Universidad Nacional Micaela Bastidas de Apurimac, Patibamba baja, Abancay, Apurimac, Perú.

Correo electrónico:oegomezq@hotmail.com 